CUBO A Mathematical Journal Vol.12, $N^{\underline{O}} 01$, (41-58). March 2010

\title{
Korteweg-de Vries-Burgers Equation on a Segment
}

\author{
ELENA I. KAIKINA \\ Instituto de Matemáticas, \\ UNAM Campus Morelia, AP 61-3 (Xangari), \\ Morelia CP 58089, Michoacán, MEXICO \\ email: ekaikina@matmor.unam.mx \\ AND \\ Leonardo Guardado-Zavala, Hector F. Ruiz-Paredes, \\ S. JuAREZ Zirate \\ Posgrado Electrica, Instituto Tecnológico de Morelia, \\ CP 58120, Morelia, Michoacán, MÉXICO \\ emails: guardado@ps.itm.mx \\ hruiz@sirio.tsemor.mx \\ sjzirate@matmor.unam.mx
}

\begin{abstract}
We study the following initial-boundary value problem for the Korteweg-de Vries-Burgers equation on the interval $(0,1)$

$$
\left\{\begin{array}{c}
u_{t}+u u_{x}-u_{x x}+u_{x x x}=0, t>0, x \in(0,1) \\
u(x, 0)=u_{0}(x), x \in(0,1) \\
u(0, t)=u(1, t)=u_{x}(1, t)=0, t>0 .
\end{array}\right.
$$

We prove that if the initial data $u_{0} \in \mathbf{L}^{2}$, then there exists a unique solution $u \in \mathbf{C}\left([0, \infty) ; \mathbf{L}^{2}\right) \cup$ $\mathbf{C}\left((0, \infty) ; \mathbf{H}^{1}\right)$ of the initial-boundary value problem $(0.1)$. We also obtain the large time asymptotic of solution uniformly with respect to $x \in(0,1)$ as $t \rightarrow \infty$.
\end{abstract}




\section{RESUMEN}

Estudiamos el siguiente problema de valor inicial en la frontera para la ecuación de Kortewegde Vries-Burgers en el intervalo $(0,1)$

$$
\left\{\begin{array}{c}
u_{t}+u u_{x}-u_{x x}+u_{x x x}=0, t>0, x \in(0,1) \\
u(x, 0)=u_{0}(x), x \in(0,1) \\
u(0, t)=u(1, t)=u_{x}(1, t)=0, t>0 .
\end{array}\right.
$$

Provamos que si el dato inicial $u_{0} \in \mathbf{L}^{2}$, entonces existe una única solución $u \in \mathbf{C}\left([0, \infty) ; \mathbf{L}^{2}\right) \cup$ $\mathbf{C}\left((0, \infty) ; \mathbf{H}^{1}\right)$ del problema de valor inicial en la frontera (0.1). También obtenemos comportamiento asintótico de la solución con respecto a $x \in(0,1)$ cuando $t \rightarrow \infty$.

Key words and phrases: Dissipative Nonlinear Evolution Equation, Large Time Asymptotics, Korteweg-de Vries-Burgers equation.

Math. Subj. Class.: 35Q35.

\section{Introduction}

We study the global existence and large time asymptotic behavior of solutions to the initialboundary value problem for the Korteweg-de Vries-Burgers equation in the interval $(0,1)$

$$
\left\{\begin{array}{c}
u_{t}+u u_{x}-u_{x x}+u_{x x x}=0, t>0, x \in(0,1) \\
u(x, 0)=u_{0}(x), x \in(0,1) \\
u(0, t)=u(1, t)=u_{x}(1, t)=0, t>0
\end{array}\right.
$$

The Korteweg-de Vries-Burgers equation (1.1) is a simple universal model equation which appears as the first approximation in the description of the dispersive-dissipative nonlinear waves, and has many applications in various fields of Physics, Biology and Engineering. In the case of the Cauchy problem some estimates for the time decay rates of solutions to the Korteweg-de VriesBurgers type equations were found in papers [3], [4], [5] and the large time asymptotics of solutions was obtained in [6], [11], [12]. In the case of the boundary value problem on half-line the large time asymptotics of solutions were studied in papers [2], [7], [8],[9], [10]. As far as we know large time asymptotic behavior for solutions of the initial-boundary value problem for the Korteweg-de Vries-Burgers equation (1.1) on the interval was not studied previously. In this paper we consider (1.1) in the case of the initial data belonging to $\mathbf{L}^{2}$. We note here that we do not assume the smallness condition on the data. In the case of large initial data it is more difficult than that of small data to obtain exact representation of large time asymptotics of solutions and there are a few results (see, e.g. [13]). Another difficulty in the study of the boundary value problem for the Korteweg-de Vries-Burgers equation (1.1) is that the linear operator $-\partial_{x}^{2}+\partial_{x}^{3}$ is not self-adjoint 
and we can not apply the Fourier method when we take the boundary value into account. To avoid this difficulty we apply the Laplace transformation with respect to space variable to derive the Green function of the resulting equation. For obtaining $\mathbf{L}^{p}$-estimates of the Green function we use the method of papers [8] and [9].

To state the results of the present paper precisely we give some notations. Let us denote $\mathbf{H}^{1}=\left\{\varphi \in \mathbf{L}^{2}(0,1) ;\|\varphi\|_{\mathbf{H}^{1}}=\|\varphi\|_{\mathbf{L}^{2}}+\left\|\varphi_{x}\right\|_{\mathbf{L}^{2}}<\infty\right\}$.

We introduce the function $\Lambda(x) \in \mathbf{L}^{\infty}(0,1)$

$$
\begin{aligned}
\Lambda(x) & =-\frac{\widetilde{\triangle}\left(-\xi_{0}, 1-x\right)}{\widetilde{\triangle}^{\prime}\left(-\xi_{0}, 1\right)}, \widetilde{\triangle}(\xi, y)=\sum_{j=1}^{3} e^{-\phi_{j} y} \phi_{j}^{\prime}(\xi), \\
\widetilde{\triangle}^{\prime}(\xi, 1) & =\sum_{j=1}^{3} e^{-\phi_{j} y}\left(\phi_{j}^{\prime \prime}(\xi)-\left(\phi_{j}^{\prime}(\xi)\right)^{2}\right),
\end{aligned}
$$

where

$$
\begin{aligned}
\widetilde{\triangle}(\xi, y) & =\sum_{j=1}^{3} e^{-\phi_{j} y} \phi_{j}^{\prime}(\xi) \\
\widetilde{\triangle}^{\prime}(\xi, y) & =\sum_{j=1}^{3} e^{-\phi_{j} y}\left(\phi_{j}^{\prime \prime}(\xi)-\left(\phi_{j}^{\prime}(\xi)\right)^{2}\right) .
\end{aligned}
$$

Here $\phi_{l}(\xi)$ are the roots of the characteristic equation $-p^{2}+p^{3}+\xi=0$, such that $\operatorname{Re} \phi_{l}(\xi)>0$, $l=1,2$, and $\operatorname{Re} \phi_{3}(\xi)<0$, for all

$$
\xi \in D_{0}=\left\{\xi \in \mathbf{C}: \operatorname{Re} \xi \geq 0, \xi \notin\left[0, \frac{4}{27}\right]\right\},
$$

and $\xi_{0} \in \mathbb{C}, \operatorname{Re} \xi_{0}>0$ is the first root of the equation

$$
\sum_{j=1}^{3} e^{-\phi_{j}} \phi_{j}^{\prime}(-\xi)=0 .
$$

By the same letter $C$ we denote different positive constants if it does not make confusion.

We state the main result of this paper.

Theorem 1.1. Suppose that the initial data $u_{0} \in \mathbf{L}^{2}$. Then there exists a unique solution of (1.1)

$$
u \in \mathbf{C}\left([0, \infty) ; \mathbf{L}^{2}\right) \cup \mathbf{C}\left((0, \infty) ; \mathbf{H}^{1}\right)
$$

such that the solution has the following asymptotics

$$
u(x, t)=A \Lambda(x) e^{-\xi_{0} t}+O\left(e^{-\left(\xi_{0}+\delta\right) t}\right)
$$

for $t \rightarrow \infty$ uniformly with respect to $x \in(0,1)$, where $\delta>0$ is a constant satisfying the condition such that $\left|\xi_{0}\right|+\delta<\left|\xi_{1}\right|$, where $\xi_{1}$ is the second root of (1.2), the constant $A$ is defined by 


$$
A=\int_{0}^{1} u_{0}(y) \widetilde{\triangle}\left(-\xi_{0}, y\right) d y+\int_{0}^{\infty} e^{\xi_{0} \tau} d \tau \int_{0}^{1} u(y, \tau) u_{y}(y, \tau) \widetilde{\triangle}\left(-\xi_{0}, y\right) d y
$$

Remark 1.1. By virtue of the numerical computations via program Maple we have $\xi_{0} \approx 70$ and $\xi_{1} \approx 200$.

We organize our paper as follows. In Section 2 we solve the linear initial-boundary value problem corresponding to (1.1). In Section 3 we prove the local existence of solutions to (1.1). Section 4 is devoted to the proof of global existence of solutions to (1.1) for the case of small initial data. We prove Theorem 1.1 in Section 5 by using the time decay estimates of solutions obtained in Section 4.

\section{Linear Problem}

We consider the following linear initial-boundary value problem

$$
\left\{\begin{array}{c}
u_{t}-u_{x x}+u_{x x x}=f, \quad t>0, x \in(0,1), \\
u(x, 0)=u_{0}(x), \quad x \in(0,1) \\
u(0, t)=u(1, t)=u_{x}(1, t)=0, t>0 .
\end{array}\right.
$$

We define for $x \in(0,1)$

$$
\widetilde{\triangle}(\xi, x)=\sum_{j=1}^{3} e^{-\phi_{j} x} \phi_{j}^{\prime}(\xi),
$$

where $\phi_{l}(\xi)$ are the roots of the characteristic equation $-p^{2}+p^{3}+\xi=0$, such that $\operatorname{Re} \phi_{l}(\xi)>0$, $l=1,2$, and $\operatorname{Re} \phi_{3}(\xi)<0$, for all

$$
\xi \in D_{0}=\left\{\xi \in \mathbf{C}: \operatorname{Re} \xi \geq 0, \xi \notin\left[0, \frac{4}{27}\right]\right\}
$$

Denote by

$$
\mathcal{G} f=\Theta(x)\left(\int_{0}^{x} f(y) F_{1}(x, y, t) d y+\int_{x}^{1} f(y) F_{2}(x, y, t) d y\right)
$$

where

$$
\begin{gathered}
\Theta(x)=\left\{\begin{array}{c}
1, x \in[0,1] \\
0, x \notin[0,1],
\end{array}\right. \\
F_{1}(x, y, t)=-\frac{1}{2 \pi i} \int_{-i \infty}^{i \infty} e^{\xi t} \frac{1}{\widetilde{\triangle}(\xi, 1)} \widetilde{\triangle}(\xi, 1-x) \widetilde{\triangle}(\xi, y) d \xi
\end{gathered}
$$

and

$$
F_{2}(x, y, t)=-\frac{1}{2 \pi i} \int_{-i \infty}^{i \infty} d \xi e^{\xi t} \frac{\widetilde{\triangle}(\xi, 1-x) \widetilde{\triangle}(\xi, y)-\widetilde{\triangle}(\xi, y-x) \widetilde{\triangle}(\xi, 1)}{\widetilde{\triangle}(\xi, 1)}
$$


Proposition 2.1. Let the initial data $u_{0} \in \mathbf{L}^{2}$ and $f \in \mathbf{L}^{2}$ Then a solution $u$ of (2.1) has the following representation

$$
u(x, t)=\mathcal{G} u_{0}+\int_{0}^{t} \mathcal{G}(t-\tau) f(\tau) d \tau .
$$

Proof. To derive an integral representation for solutions of the problem (2.1) we suppose that there exists a solution $u(x, t)$ of problem (2.1), which is continued by zero outside of $x<0, x>1$

$$
\begin{aligned}
u(x, t) & =0 \text { for all } x \notin[0,1], \\
\partial_{x}^{j} u(0, t) & =\lim _{x \rightarrow 0+} \partial_{x}^{j} u(x, t), j=0,1,2 \\
\partial_{x}^{j} u(1, t) & =\lim _{x \rightarrow 1-} \partial_{x}^{j} u(x, t), j=0,1,2 .
\end{aligned}
$$

Also we suppose that $f=0$. We denote operator

$$
\mathbb{P}[\phi(p, t)]=-\frac{1}{2 \pi i} \int_{-i \infty}^{i \infty} \frac{e^{(q-p)}-1}{q-p} \phi(q, t) d q .
$$

We have for the Laplace transform

$$
\mathcal{L}\{\mathbb{K} u\}=\mathbb{P}\left[K(p) \widehat{u}+p^{2} \sum_{j=1}^{2} \frac{\partial_{x}^{j-1} u(0, t)-e^{-p} \partial_{x}^{j-1} u(a, t)}{p^{j}}-p^{3} \sum_{j=1}^{3} \frac{\partial_{x}^{j-1} u(0, t)-e^{-p} \partial_{x}^{j-1} u(a, t)}{p^{j}}\right] .
$$

Since $\mathcal{L}\{u\}$ is analytic for all $p \in \mathbb{C}$ we have

$$
\mathcal{L}\{u\}=\widehat{u}(p, t)=\mathbb{P}[\widehat{u}(p, t)] .
$$

Applying the Laplace transform with respect to $x$ to problem (2.1) we obtain

$$
\left\{\begin{aligned}
& \mathbb{P}\left[\widehat{u}_{t}+K(p) \widehat{u}(p, t)+\right.\left.B_{1}(p, t)-e^{-p} B_{2}(p, t)\right]=0, t>0, x>0, \\
& \widehat{u}(p, 0)=\widehat{u}_{0}(p), \\
& u(0, t)=u(1, t)=u_{x}(0, t)=0, t>0
\end{aligned}\right.
$$

where

$$
\begin{aligned}
& B_{1}(p, t)=p^{2} \sum_{j=1}^{2} \frac{\partial_{x}^{j-1} u(0, t)}{p^{j}}-p^{3} \sum_{j=1}^{3} \frac{\partial_{x}^{j-1} u(0, t)}{p^{j}} \\
& B_{2}(p, t)=p^{2} \sum_{j=1}^{2} \frac{\partial_{x}^{j-1} u(a, t)}{p^{j}}-p^{3} \sum_{j=1}^{2} \frac{\partial_{x}^{j-1} u(a, t)}{p^{j}} .
\end{aligned}
$$

We rewrite (2.4) in the form

$$
\widehat{u}_{t}+K(p) \widehat{u}(p, t)+B_{1}(p, t)-e^{-p} B_{2}(p, t)=\Phi(p, t),
$$


where some function $\Phi(p, t)$ is analytic for all $p \in \mathbb{C}$,

$$
|\Phi(p, t)| \leq C(t) \frac{1+\left|e^{-p}\right|}{|p|},|p|>1
$$

and

$$
\mathbb{P}[\Phi(p, t)]=0
$$

Now we prove that under this conditions $\Phi(p, t) \equiv 0$.To find $\Phi(p, t)$ we introduce functions

$$
\begin{aligned}
& \Omega_{1}(z, t)=\frac{1}{2 \pi i} \int_{-i \infty}^{i \infty} \frac{1}{q-z} \Phi(q, t) d q \\
& \Omega_{2}(z, t)=\frac{e^{-z}}{2 \pi i} \int_{-i \infty}^{i \infty} \frac{e^{q}}{q-z} \Phi(q, t) d q .
\end{aligned}
$$

Since $\Phi(p, t)$ satisfies Holder condition the functions $\Omega_{1}(z, \xi), \Omega_{2}(z, \xi)$ are analytic in $\operatorname{Re} z \neq 0$. Denote by

$$
\Omega_{1,2}^{+}(p, t)=\lim _{z \rightarrow p, \operatorname{Re} z<0} \Omega_{1,2}(z, t)
$$

and

$$
\Omega_{1,2}^{-}(p, t)=\lim _{z \rightarrow p, \operatorname{Re} z>0} \Omega_{1,2}(z, t) .
$$

for $\operatorname{Re} p=0$.Since function $\Phi(p, t)$ is analytic for all $p \in \mathbb{C}$ from estimate (2.7) we have

$$
\Omega_{2}^{-}(p, \xi)=\Omega_{1}^{+}(p, \xi)=0
$$

Another hand by Sokhotsky-Plemeli formula we get

$$
\begin{aligned}
& \Omega_{2}^{-}(p, \xi)=V P \frac{e^{-p}}{2 \pi i} \int_{-i \infty}^{i \infty} \frac{1}{q-p} \Phi(p, t) d q-\frac{1}{2} \Phi(p, t) \\
& \Omega_{1}^{+}(p, \xi)=V P \frac{1}{2 \pi i} \int_{-i \infty}^{i \infty} \frac{1}{q-p} \Phi(p, t) d q+\frac{1}{2} \Phi(p, t) .
\end{aligned}
$$

and therefore for $\operatorname{Re} p=0$

$$
\Omega_{2}^{-}(p, \xi)-\Omega_{1}^{+}(p, \xi)=\mathbb{P}[\Phi(p, t)]-\Phi(p, t)=0
$$

Thus for $\operatorname{Re} p=0$

$$
\Phi(p, t)=\mathbb{P}[\Phi(p, t)]=0
$$

and therefore due to analycity $\Phi(p, t) \equiv 0$ for all $p \in \mathbb{C}$.

Applying the Laplace transformation to problem (2.6) with respect to time variable we write $\mathcal{L}_{t}\{\widehat{u}(p, t)\}=\widehat{\widehat{u}}(p, \xi)$ as

$$
\widehat{\widehat{u}}(p, \xi)=\frac{1}{K(p)+\xi}\left(\widehat{u}_{0}(p)-\widehat{B}_{1}(p, \xi)+e^{-p} \widehat{B}_{2}(p, \xi)\right)
$$


for $p \in \mathbb{C}, K(p)=-p^{2}+p^{3}$.

Here functions $\widehat{B}_{1}(p, \xi), \widehat{B}_{2}(p, \xi)$ are the Laplace transforms of $B_{1}(p, t), B_{2}(p, t)$ with respect to time.

In order to get the integral formula for solution, we need to know the functions $\widehat{B}_{1}(p, \xi)$, $\widehat{B}_{2}(p, \xi)$. We will find its using the analytic condition of function $\widehat{\widehat{u}}$ for $p \in \mathbb{C}$ and $\operatorname{Re} \xi>0$

$$
\widehat{\widehat{u}}(p, \xi)=\mathbb{P}\{\widehat{\widehat{u}}(p, \xi)\} .
$$

Via (2.9) we rewrite (2.10) in the form

$$
\begin{aligned}
& \frac{1}{K(p)+\xi}\left(\widehat{u}_{0}(p)-\widehat{B}_{1}(p, \xi)+e^{-p} \widehat{B}_{2}(p, \xi)\right) \\
= & \frac{1}{2 \pi i} \int_{-i \infty}^{i \infty} \frac{e^{(q-p)}-1}{q-p} \frac{1}{K(q)+\xi}\left(\widehat{u}_{0}(q)-\widehat{B}_{1}(q, \xi)+e^{-p} \widehat{B}_{2}(q, \xi)\right) .
\end{aligned}
$$

Let $\phi_{l}(\xi)$ are the roots of the characteristic equation $-p^{2}+p^{3}+\xi=0$, such that $\operatorname{Re} \phi_{l}(\xi)>0$, $l=1,2$, and $\operatorname{Re} \phi_{3}(\xi)<0$, for all $\xi \in D_{0}=\left\{\xi \in \mathbf{C}: \operatorname{Re} \xi \geq 0, \xi \notin\left[0, \frac{4}{27}\right]\right\}$. Note that the functions $\phi_{l}(\xi)$ are analytic in the domain $\left\{\xi \in \mathbf{C}: \xi \notin\left(-\infty, \frac{4}{27}\right]\right\}$. We represent $p^{2}=\frac{\xi}{1-p}$ for $|p|<1$ and $p^{3}=\frac{-\xi}{1-\frac{1}{p}}$ for $|p|>1$, hence we get the asymptotics

$$
\begin{gathered}
\phi_{1}(\xi)=\left\{\begin{array}{c}
\sqrt{\xi}+O(|\xi|), \xi \rightarrow 0, \operatorname{Im} \xi>0,1+O(|\xi|), \xi \rightarrow 0, \operatorname{Im} \xi<0, \\
e^{i \frac{\pi}{3}} \sqrt[3]{\xi}+O(1),|\xi| \rightarrow \infty,
\end{array}\right. \\
\phi_{2}(\xi)=\left\{\begin{array}{c}
1+O(|\xi|), \xi \rightarrow 0, \operatorname{Im} \xi>0, \sqrt{\xi}+O(|\xi|), \xi \rightarrow 0, \operatorname{Im} \xi<0, \\
e^{-i \frac{\pi}{3}} \sqrt[3]{\xi}+O(1),|\xi| \rightarrow \infty,
\end{array}\right.
\end{gathered}
$$

and

$$
\phi_{3}(\xi)=\left\{\begin{array}{l}
-\sqrt{\xi}+O(|\xi|),|\xi| \rightarrow 0, \\
-\sqrt[3]{\xi}+O(1),|\xi| \rightarrow \infty,
\end{array}\right.
$$

for all $\xi \in \mathbf{C}: \xi \notin\left(-\infty, \frac{4}{27}\right]$ (by $\sqrt{\xi}$ and $\sqrt[3]{\xi}$ we denote the main value of the analytic function, i.e. $\sqrt{1}=\sqrt[3]{1}=1$.)

By Cauchy Theorem we have for all $p \in \mathbb{C}$

$$
\begin{aligned}
& \frac{1}{2 \pi i} \int_{-i \infty}^{i \infty} \frac{e^{(q-p)}-1}{q-p} \frac{1}{K(q)+\xi}\left(\widehat{u}_{0}(q)-\widehat{B}_{1}(q, \xi)+e^{-p} \widehat{B}_{2}(q, \xi)\right) \\
= & \frac{1}{K(p)+\xi}\left(\widehat{u}_{0}(p)-\widehat{B}_{1}(p, \xi)+e^{-p} \widehat{B}_{2}(p, \xi)\right) \\
& +\frac{e^{\left(\phi_{3}(\xi)-p\right)}}{\phi_{3}-p} \phi_{3}^{\prime}(\xi)\left(-\widehat{u}_{0}\left(\phi_{3}\right)+\widehat{B}_{1}\left(\phi_{3}, \xi\right)-e^{-\phi_{3}} \widehat{B}_{2}\left(\phi_{3}, \xi\right)\right) \\
& -\sum_{j=1}^{2} \frac{1}{\phi_{j}-p} \phi_{j}^{\prime}(\xi)\left(\widehat{u}_{0}\left(\phi_{j}\right)-\widehat{B}_{1}\left(\phi_{j}, \xi\right)+e^{-\phi_{j}} \widehat{B}_{2}\left(\phi_{j}, \xi\right)\right) .
\end{aligned}
$$


Using (2.11) we get

$$
\left\{\begin{array}{c}
\widehat{B}_{2}\left(\phi_{3}, \xi\right)=e^{\phi_{3}}\left(-\widehat{u}_{0}\left(\phi_{3}\right)+\widehat{B}_{1}\left(\phi_{3}, \xi\right)\right) \\
\widehat{B}_{1}\left(\phi_{j}, \xi\right)=\widehat{u}_{0}\left(\phi_{j}\right)+e^{-\phi_{j}} \widehat{B}_{2}\left(\phi_{j}, \xi\right), j=1,2
\end{array}\right.
$$

So we need to put in the initial-boundary value problem one boundary data in the point $x=0$ and two boundary data in the point $x=1$.

Let, for example, $u(0, t)=u_{x}(1, t)=u(1, t)=0$. Thus from system $(2.16)$ we get

$$
\left\{\begin{array}{c}
-\partial_{x x} \widehat{u}(a, \xi)=e^{\phi_{3}(\xi) a}\left(-\widehat{u}_{0}\left(\phi_{3}\right)+\partial_{x} \widehat{u}(0, \xi)\left(1-\phi_{3}\right)-\partial_{x x} \widehat{u}(0, \xi)\right) \\
\partial_{x} \widehat{u}(0, \xi)\left(1-\phi_{j}\right)-\partial_{x x} \widehat{u}(0, \xi)=\widehat{u}_{0}\left(\phi_{j}\right)-e^{-\phi_{j} a} \partial_{x x} \widehat{u}(a, \xi), j=1,2,
\end{array}\right.
$$

which is equal to

$$
\left(\begin{array}{ccc}
e^{-\phi_{1}(\xi)} & 1-\phi_{1} & -1 \\
e^{-\phi_{2}(\xi)} & 1-\phi_{2} & -1 \\
e^{-\phi_{3}(\xi)} & 1-\phi_{3} & -1
\end{array}\right)\left(\begin{array}{c}
\partial_{x x} \widehat{u}(1, \xi) \\
\partial_{x} \widehat{u}(0, \xi) \\
\partial_{x x} \widehat{u}(0, \xi)
\end{array}\right)=\left(\begin{array}{c}
\widehat{u}_{0}\left(\phi_{1}\right) \\
\widehat{u}_{0}\left(\phi_{2}\right) \\
\widehat{u}_{0}\left(\phi_{3}\right)
\end{array}\right)
$$

Denote the determinant of this system by $\triangle\left(\phi_{1}, \phi_{2}, \phi_{3}\right)$, then it has a form

$$
\begin{aligned}
\triangle\left(\phi_{1}, \phi_{2}, \phi_{3}\right) & =\left|\begin{array}{lll}
1 & 1 & 1 \\
e^{-\phi_{1}(\xi)} & e^{-\phi_{2}(\xi)} & e^{\phi_{3}(\xi)} \\
\phi_{1}(\xi) e^{\phi_{1}(\xi)} & \phi_{2}(\xi) e^{\phi_{2}(\xi)} & \phi_{3}(\xi) e^{\phi_{3}(\xi)}
\end{array}\right| \\
& =e^{-\phi_{1}}\left(\phi_{2}-\phi_{3}\right)+e^{-\phi_{2}}\left(\phi_{3}-\phi_{1}\right)+e^{-\phi_{3}}\left(\phi_{1}-\phi_{2}\right) .
\end{aligned}
$$

in the domain $\xi \in D_{0}$.

Since $\sum_{j=1}^{3} \phi_{j}=1$ and

$$
\begin{aligned}
\phi_{1}^{\prime}(\xi) & =-\frac{1}{\left(\phi_{1}-\phi_{2}\right)\left(\phi_{1}-\phi_{3}\right)} ; \phi_{2}^{\prime}(\xi)=-\frac{1}{\left(\phi_{2}-\phi_{1}\right)\left(\phi_{2}-\phi_{3}\right)} \\
\phi_{3}^{\prime}(\xi) & =-\frac{1}{\left(\phi_{3}-\phi_{1}\right)\left(\phi_{3}-\phi_{2}\right)}
\end{aligned}
$$

we can rewrite $\triangle\left(\phi_{1}, \phi_{2}, \phi_{3}\right)$ as

$$
\triangle\left(\phi_{1}, \phi_{2}, \phi_{3}\right)=V(\xi) \sum_{j=1}^{3} e^{-\phi_{j}(\xi)} \phi_{j}^{\prime}(\xi)
$$

where

$$
V(\xi)=\left(\phi_{1}-\phi_{2}\right)\left(\phi_{2}-\phi_{3}\right)\left(\phi_{3}-\phi_{1}\right)
$$

Since $V(\xi) \neq 0$ and $\operatorname{Re} \phi_{l}(\xi)>0, l=1,2, \operatorname{Re} \phi_{3}(\xi)<0$ in domain $\xi \in D_{0}$ we easily get for $|\xi| \gg 1, \xi \in D_{0}$

$$
\triangle\left(\phi_{1}, \phi_{2}, \phi_{3}\right) \neq 0 .
$$


By numeric computations we can check that $\triangle\left(\phi_{1}, \phi_{2}, \phi_{3}\right) \neq 0$ for all $|\xi| \leq C$, $\xi \in D_{0}=\left\{\xi \in \mathbf{C}: \operatorname{Re} \xi \geq 0, \xi \notin\left[0, \frac{4}{27}\right]\right\}$. Therefore there exists a unique solution of the system (2.17)

$$
\left(\begin{array}{c}
\partial_{x x} \widehat{u}(1, \xi) \\
\partial_{x} \widehat{u}(0, \xi) \\
\partial_{x x} \widehat{u}(0, \xi)
\end{array}\right)=\int_{0}^{1} d y u_{0}(y)\left(\begin{array}{ccc}
e^{-\phi_{1}(\xi)} & 1-\phi_{1} & -1 \\
e^{-\phi_{2}(\xi)} & 1-\phi_{2} & -1 \\
e^{-\phi_{3}(\xi)} & 1-\phi_{3} & -1
\end{array}\right)^{-1}\left(\begin{array}{c}
e^{-\phi_{1} y} \\
e^{-\phi_{2} y} \\
e^{-\phi_{3} y}
\end{array}\right) .
$$

Since $u(0, t)=u_{x}(1, t)=u(1, t)=0$ by $(2.9)$ and $(2.5)$ we have

$$
\left.\widehat{\widehat{u}}(p, \xi)=\frac{1}{K(p)+\xi}\left(\widehat{u}_{0}(p)+(p-1) \widehat{u}_{x}(0, \xi)\right)+\widehat{u}_{x x}(0, \xi)-e^{-p a} \widehat{u}_{x x}(a, \xi)\right) .
$$

Taking inverse Laplace transform with respect to variable $p$ we get

$$
\begin{aligned}
\hat{u}(x, \xi)= & \Theta(x) \int_{0}^{1} d y u_{0}(y) \frac{1}{2 \pi i} \int_{-i \infty}^{+i \infty} d p e^{p x} \frac{1}{K(p)+\xi} \\
& \left.\times\left(e^{-p y}+(p-1) \widehat{u}_{x}(0, \xi)\right)+\widehat{u}_{x x}(0, \xi)-e^{-p} \widehat{u}_{x x}(a, \xi)\right) .
\end{aligned}
$$

By the method of residues we see that for $\xi \in D_{0}$

$$
\hat{u}(x, \xi)=\Theta(x) \int_{0}^{x} d y u_{0}(y) \widehat{F}_{1}(x, y, \xi)+\int_{x}^{1} d y u_{0}(y) \widehat{F}_{2}(x, y, \xi),
$$

where

$$
\begin{aligned}
F_{1}(x, y, \xi)= & -\phi_{3}^{\prime} e^{\phi_{3}(\xi) x}\left(e^{-y \phi_{3}(\xi)}+\left(\phi_{3}-1\right) \widehat{u}_{x}(0, \xi)+\widehat{u}_{x x}(0, \xi)\right) \\
& -\sum_{j=1}^{2} \phi_{j}^{\prime}(\xi) e^{-\phi_{j}(1-x)} \widehat{u}_{x x}(1, \xi)
\end{aligned}
$$

and

$$
\begin{aligned}
F_{2}(x, y, \xi)= & -\phi_{3}^{\prime} e^{\phi_{3}(\xi) x}\left(\left(\phi_{3}-1\right) \widehat{u}_{x}(0, \xi)+\widehat{u}_{x x}(0, \xi)\right) \\
& -\sum_{j=1}^{2} \phi_{j}^{\prime}(\xi)\left(e^{-\phi_{j}(1-x)} \widehat{u}_{x x}(1, \xi)-e^{-\phi_{j}(\xi)(x-y)}\right) .
\end{aligned}
$$

Denote $\widetilde{\triangle}(\xi, y)=\sum_{j=1}^{3} e^{-\phi_{j} y} \phi_{j}^{\prime}(\xi)$.

Substituting (2.20) into (2.23) and (2.24) and using (2.18) we get

$$
\begin{aligned}
\widehat{F}_{1}(x, y, \xi) & =-\frac{\widetilde{\triangle}(\xi, 1-x) \widetilde{\triangle}(\xi, y)}{\widetilde{\triangle}(\xi, 1)} \\
\widehat{F}_{2}(x, y, \xi) & =\frac{\widetilde{\triangle}(\xi, y-x) \widetilde{\triangle}(\xi, 1)-\widetilde{\triangle}(\xi, 1-x) \widetilde{\triangle}(\xi, y)}{\widetilde{\triangle}(\xi, 1)} .
\end{aligned}
$$


Since $\phi_{l}^{\prime}(\xi)=O\left(|\xi|^{-\frac{1}{2}}\right), l=1,2,3$ for $|\xi|<1, \xi \in D_{0}$, we have

$$
\frac{\widetilde{\triangle}(\xi, 1-x) \widetilde{\triangle}(\xi, y)}{\widetilde{\triangle}(\xi, 1)}=\frac{\left(\sum_{j=1}^{3} e^{-\phi_{j}(\xi)(1-x)} \phi_{j}^{\prime}\right)\left(\sum_{j=1}^{3} e^{-\phi_{j}(\xi) y} \phi_{j}^{\prime}\right)}{\sum_{j=1}^{3} e^{-\phi_{j}(\xi)} \phi_{j}^{\prime}}=O\left(|\xi|^{-\frac{1}{2}}\right)
$$

and

$$
\widetilde{\triangle}(\xi, y-x)=O\left(|\xi|^{-\frac{1}{2}}\right)
$$

for $|\xi|<1, \xi \in D_{0}$. Due to the fact that $\operatorname{Re} \phi_{l}(\xi)>0, l=1,2, \operatorname{Re} \phi_{3}(\xi)<0$ for $|\xi|>1, \xi \in D_{0}$, we obtain for $|\xi|>1$

$$
\begin{aligned}
& \frac{1}{\widetilde{\triangle}(\xi, 1)} \widetilde{\triangle}(\xi, 1-x) \widetilde{\triangle}(\xi, y) \\
& =\frac{e^{-\phi_{3}(1-x+y)}\left(\phi_{3}^{\prime}\right)^{2}\left(1+\sum_{i=1}^{2} O\left(e^{\left(-\phi_{j}+\phi_{3}\right)(1-x)} \frac{\phi_{j}^{\prime}}{\phi_{3}^{\prime}}\right)\right)}{e^{-\phi_{3} \phi_{3}^{\prime}}\left(1+\sum_{j=1}^{2} O\left(e^{\left(-\phi_{j}+\phi_{3}\right)} \frac{\phi_{j}^{\prime}}{\phi_{3}^{\prime}}\right)\right)} \\
& \times\left(1+\sum_{j=1}^{2} O\left(e^{\left(-\phi_{j}+\phi_{3}\right) y} \frac{\phi_{j}^{\prime}}{\phi_{3}^{\prime}}\right)\right) \\
& =e^{\phi_{3}(x-y)} \phi_{3}^{\prime}\left(1+\sum_{i=1}^{2} O\left(e^{\left(-\phi_{j}+\phi_{3}\right) y}\right)+\sum_{i=1}^{2} O\left(e^{\left(-\phi_{j}+\phi_{3}\right)(1-x)}\right)\right) .
\end{aligned}
$$

Therefore taking the asymptotics (2.12)-(2.14) into account we find that

$$
\widehat{F}_{1}(x, y, \xi)=O\left(\xi^{-\frac{2}{3}} e^{-C \sqrt[3]{|\xi|}(x-y)}\right)
$$

for $\xi \in D_{0},|\xi|>1, x>y$. Also from (2.27) we get

$$
\begin{aligned}
\widehat{F}_{2}(x, y, \xi)= & \widetilde{\triangle}(\xi, y-x)-\frac{\widetilde{\triangle}(\xi, 1-x) \widetilde{\triangle}(\xi, y)}{\widetilde{\triangle}(\xi, 1)}=\sum_{j=1}^{2}\left(\phi_{j}^{\prime} e^{-\phi_{j}(y-x)}\right. \\
& \left.+O\left(\phi_{3}^{\prime} e^{-\operatorname{Re} \phi_{j} y+\operatorname{Re} \phi_{3} x}\right)+O\left(\phi_{3}^{\prime} e^{-\operatorname{Re} \phi_{j}(1-x)+\operatorname{Re} \phi_{3}(1-y)}\right)\right) \\
= & O\left(\xi^{-\frac{2}{3}} e^{-C \sqrt[3]{|\xi|}(y-x)}\right)
\end{aligned}
$$

for $\xi \in D_{0},|\xi|>1, y>x$. Thus there exist the inverse Laplace transforms for the functions $\widehat{F}_{1}(x, y, \xi)$ and $\widehat{F}_{2}(x, y, \xi)$. Taking the inverse Laplace transformation of (2.23) and (2.24) we obtain

$$
F_{1}(x, y, t)=\mathcal{L}^{-1}\left\{\widehat{F}_{1}(x, y, \xi)\right\}=-\frac{1}{2 \pi i} \int_{\Gamma_{0}} e^{\xi t} \frac{1}{\widetilde{\triangle}(\xi, 1)} \widetilde{\triangle}(\xi, 1-x) \widetilde{\triangle}(\xi, y) d \xi
$$

and

$$
F_{2}(x, y, t)=\mathcal{L}^{-1}\left\{\widehat{F}_{1}(x, y, \xi)\right\}=-\frac{1}{2 \pi i} \int_{\Gamma_{0}} d \xi e^{\xi t} \frac{\widetilde{\triangle}(\xi, 1-x) \widetilde{\triangle}(\xi, y)-\widetilde{\triangle}(\xi, y-x) \widetilde{\triangle}(\xi, 1)}{\widetilde{\triangle}(\xi, 1)},
$$


where $\Gamma_{0}=\partial D_{0}$, i.e.

$$
\Gamma_{0}=(-i \infty,-i 0) \cup\left[-i 0, \frac{4}{27}-i 0\right] \cup\left[\frac{4}{27}+i 0, i 0\right] \cup(i 0, i \infty) .
$$

Since functions $\widehat{F}_{l}(x, y, \xi)$ are symmetric with respect to $\phi_{1}, \phi_{2}, \phi_{3}$, using the relations $\overline{\phi_{1}(\xi)}=$ $\phi_{2}(\bar{\xi}), \overline{\phi_{2}(\xi)}=\phi_{1}(\bar{\xi}), \overline{\phi_{3}(\xi)}=\phi_{3}(\bar{\xi})$ for all $\xi \in D_{0}$ we can change the contour of integration $\Gamma_{0}$ to the imaginary axis $(-i \infty, i \infty)$ to get

$$
F_{1}(x, y, t)=-\frac{1}{2 \pi i} \int_{-i \infty}^{i \infty} e^{\xi t} \frac{1}{\widetilde{\triangle}(\xi, 1)} \widetilde{\triangle}(\xi, 1-x) \widetilde{\triangle}(\xi, y) d \xi
$$

and

$$
F_{2}(x, y, t)=-\frac{1}{2 \pi i} \int_{-i \infty}^{i \infty} d \xi e^{\xi t} \frac{\widetilde{\triangle}(\xi, 1-x) \widetilde{\triangle}(\xi, y)-\widetilde{\triangle}(\xi, y-x) \widetilde{\triangle}(\xi, 1)}{\widetilde{\triangle}(\xi, 1)} .
$$

Therefore taking inverse Laplace transform of (2.22) with respect to $\xi$ we obtain

$$
u(x, t)=\mathcal{G} u_{0} .
$$

Thus by Duhamel principle Proposition is proved.

Lemma 2.2. We have the asymptotics for large time

$$
F_{j}(x, y, t)=-e^{-\xi_{0} t} \Lambda(x) \widetilde{\triangle}\left(-\xi_{0}, y\right)+O\left(e^{-\left(\xi_{0}+\delta\right) t}\right)
$$

and estimates

$$
\left|\partial_{x}^{n} F_{j}(x, y, t)\right| \leq C e^{-\xi_{0} t}\{t\}^{-\alpha}|x-y|^{2 \alpha-1-n}
$$

for $x, y \in(0,1), x \neq y, t>0$, where $\alpha \in\left[0, \frac{n+1}{2}\right], n=0,1, j=1,2$.

Proof. We consider a curve in the complex left-half plane $\operatorname{Re} \xi<0$ such that $\operatorname{Re} \phi_{1}(\xi)=0$, it is defined by the equation $(i y)^{2}-(i y)^{3}=\xi$ with $y=\operatorname{Im} \phi_{1}(\xi)$. Therefore there exists a contour

$$
\mathcal{C}_{0}=\left\{\xi \in \mathbf{C}, \operatorname{Re} \xi<0: \operatorname{Re} \xi=O\left(|\xi|^{\frac{2}{3}}\right)\right\}
$$

such that

$$
\operatorname{Re} \phi_{l}(\xi)>0, l=1,2, \operatorname{Re} \phi_{3}(\xi)<0 \text { for all } \xi \in \mathcal{C}_{0} .
$$

We also consider a contour

$$
\begin{aligned}
\mathcal{C}_{1}= & \left(-\xi_{0}-\delta-i \infty,-\xi_{0}-\delta-i 0\right) \cup\left(-\xi_{0}-\delta-i 0,-i 0\right) \\
& \cup\left(i 0,-\xi_{0}-\delta+i 0\right) \cup\left(-\xi_{0}-\delta+i 0,-\xi_{0}-\delta+i \infty\right) .
\end{aligned}
$$

Denote

$$
\mathcal{C}_{0} \cap \mathcal{C}_{1}=\left\{z_{1}, z_{2}\right\}, \operatorname{Im} z_{1}>0, \operatorname{Im} z_{2}<0, \operatorname{Re} z_{l}=-\xi_{0}-\delta, l=1,2
$$


We now define a contour $\mathcal{C}=\mathcal{C}_{2} \cup \mathcal{C}_{3}$, where

$$
\begin{gathered}
\mathcal{C}_{2}=\left\{\xi \in \mathcal{C}_{1}: \operatorname{Im} z_{2} \leq \operatorname{Im} \xi \leq \operatorname{Im} z_{1}\right\} \\
\mathcal{C}_{3}=\left\{\xi \in \mathcal{C}_{0}: \operatorname{Im} \xi>\operatorname{Im} z_{1} \text { or } \operatorname{Im} \xi<\operatorname{Im} z_{2}\right\}
\end{gathered}
$$

Note that the asymptotic formulas (2.12)-(2.14) are valid on the contour $\mathcal{C}$. In view of them we have (2.25)-(2.29) for $\xi \in \mathcal{C}$. Therefore changing the contour of integration to $\mathcal{C}$ we obtain

$$
\begin{aligned}
F_{1}(x, y, \tau)= & -\frac{1}{2 \pi i} \int_{\xi \in \mathcal{C}_{2}} e^{\xi t} \frac{1}{\widetilde{\triangle}(\xi, 1)} \widetilde{\triangle}(\xi, 1-x) \widetilde{\triangle}(\xi, y) d \xi \\
& -\frac{1}{2 \pi i} \int_{\xi \in \mathcal{C}_{3}} e^{\xi t} \frac{1}{\widetilde{\triangle}(\xi, 1)} \widetilde{\triangle}(\xi, 1-x) \widetilde{\triangle}(\xi, y) d \xi
\end{aligned}
$$

Since $\widetilde{\triangle}(x+i 0, q)=\widetilde{\triangle}(x-i 0, q)$ we get

$$
\begin{aligned}
& -\frac{1}{2 \pi i} \int_{-\xi_{0}-\delta-i 0}^{-i 0} e^{\xi t} \frac{1}{\widetilde{\triangle}(\xi, 1)} \widetilde{\triangle}(\xi, 1-x) \widetilde{\triangle}(\xi, y) d \xi \\
& -\frac{1}{2 \pi i} \int_{+i 0}^{-\xi_{0}-\delta+i 0} e^{\xi t} \frac{1}{\widetilde{\triangle}(\xi, 1)} \widetilde{\triangle}(\xi, 1-x) \widetilde{\triangle}(\xi, y) d \xi \\
= & -e^{-\xi_{0} t} \frac{\widetilde{\triangle}\left(-\xi_{0}, 1-x\right) \widetilde{\triangle}\left(-\xi_{0}, y\right)}{\widetilde{\triangle}^{\prime}\left(-\xi_{0}, 1\right)} .
\end{aligned}
$$

Also by (2.25) we have

$$
\begin{aligned}
& \frac{1}{2 \pi i} \int_{-\xi_{0}-\delta+i 0}^{z_{1}} e^{\xi t} \frac{1}{\widetilde{\triangle}(\xi, 1)} \widetilde{\triangle}(\xi, 1-x) \widetilde{\triangle}(\xi, y) d \xi \\
& +\frac{1}{2 \pi i} \int_{z_{2}}^{-\xi_{0}-\delta-i 0} e^{\xi t} \frac{1}{\widetilde{\triangle}(\xi, 1)} \widetilde{\triangle}(\xi, 1-x) \widetilde{\triangle}(\xi, y) d \xi \\
= & O\left(e^{-\left(\xi_{0}+\delta\right) t}\right) .
\end{aligned}
$$

Taking into account (2.28) we get

$$
\begin{aligned}
& \left|-\frac{1}{2 \pi i} \int_{\xi \in \mathcal{C}_{3}} e^{\xi t} \frac{1}{\widetilde{\triangle}(\xi, 1)} \widetilde{\triangle}(\xi, 1-x) \widetilde{\triangle}(\xi, y) d \xi\right| \\
< & C e^{-\left(\xi_{0}+\delta\right) t} \int_{\xi \in \mathcal{C}_{3}} e^{-C t|\xi|^{\frac{2}{3}}+t\left(\xi_{0}+\delta\right)-C|x-y||\xi|^{\frac{1}{3}}}|\xi|^{-\frac{2}{3}} d \xi \\
< & C e^{-\left(\xi_{0}+\delta\right) t} t^{-\alpha}|x-y|^{2 \alpha-1}
\end{aligned}
$$

since $C|\xi|^{\frac{2}{3}}-\left(\xi_{0}+\delta\right) \geq 0$ for $\xi \in \mathcal{C}_{3}$, where $\alpha \in\left[0, \frac{1}{2}\right]$. By (2.33)-(2.35) we have from $(2.32)$

$$
F_{1}(x, y, t)=-e^{-\xi_{0} t} \frac{\widetilde{\triangle}\left(-\xi_{0}, 1-x\right) \widetilde{\triangle}\left(-\xi_{0}, y\right)}{\widetilde{\triangle}^{\prime}\left(-\xi_{0}, 1\right)}+O\left(e^{-\left(\xi_{0}+\delta\right) t}\right)
$$


for $x, y>0, t \geq 1$, and moreover

$$
\left|F_{1}(x, y, t)\right| \leq C e^{-\xi_{0} t}\left(1+\{t\}^{-\alpha}|x-y|^{2 \alpha-1}\right)
$$

for all $x, y \in(0,1), x \neq y, t>0$, where $\alpha \in\left[0, \frac{1}{2}\right]$. Thus the result of the lemma is true for the case $n=0$. Consider the case $n=1$. In view of the asymptotic formulas (2.12)-(2.14) we get

$$
\begin{aligned}
& \frac{\partial_{x} \widetilde{\triangle}(\xi, 1-x) \widetilde{\triangle}(\xi, y)}{\widetilde{\triangle}(\xi, 1)} \\
= & \frac{\left(\sum_{j=1}^{3} e^{-\phi_{j}(\xi)(1-x)}\left(\phi_{j}^{\prime} \phi_{j}\right)\right)\left(\sum_{j=1}^{3} e^{-\phi_{j}(\xi) y} \phi_{j}^{\prime}\right)}{\sum_{j=1}^{3} e^{-\phi_{j}(\xi)} \phi_{j}^{\prime}}=O(1)
\end{aligned}
$$

and

$$
\partial_{x} \widetilde{\triangle}(\xi, y-x)=O(1)
$$

for $|\xi|<1, \xi \in \mathcal{C}_{2}$ and in the same argument as in the proof of the estimate (2.25) we get

$$
\begin{aligned}
& \frac{\partial_{x} \widetilde{\triangle}(\xi, 1-x) \widetilde{\triangle}(\xi, y)}{\widetilde{\triangle}(\xi, 1)} \\
= & e^{-\phi_{3}(y-x)} \phi_{3} \phi_{3}^{\prime}\left(1+O\left(e^{-C \sqrt[3]{|\xi|} y}\right)+O\left(e^{-C \sqrt[3]{|\xi|}(1-x)}\right)\right)
\end{aligned}
$$

and

$$
\partial_{x} \widetilde{\triangle}(\xi, y-x)=e^{-\phi_{3}(y-x)} \phi_{3} \phi_{3}^{\prime}\left(1+O\left(e^{-C \sqrt[3]{|\xi|} y}\right)+O\left(e^{-C \sqrt[3]{|\xi|}(1-x)}\right)\right)
$$

for all $|\xi|>1, \xi \in \mathcal{C}_{3}$. Hence by the similar way to (2.33)-(2.35) we get

$$
\begin{aligned}
\left|\partial_{x} F_{1}(x, y, t)\right| \leq & e^{-\xi_{0} t}\left|\frac{\widetilde{\triangle}\left(-\xi_{0}, y\right) \partial_{x} \widetilde{\triangle}\left(-\xi_{0}, 1-x\right)}{\widetilde{\triangle}^{\prime}\left(-\xi_{0}, 1\right)}\right|+C e^{-\xi_{0} t} \\
& +C e^{-\left(\xi_{0}+\delta\right) t} \int_{\xi \in \mathcal{C}_{3}} e^{-C t|\xi|^{\frac{2}{3}}+t\left(\xi_{0}+\delta\right)-C|x-y||\xi|^{\frac{1}{3}}}|\xi|^{-\frac{1}{3}} d \xi \\
\leq & e^{-\xi_{0} t}\left(C+C\{t\}^{-\alpha}|x-y|^{2 \alpha-2}\right)
\end{aligned}
$$

for all $x, y \in(0,1), x \neq y, t>0$, where $\alpha \in[0,1]$. The function $F_{2}(x, y, t)$ is considered in the same way. Lemma 2.2 is proved.

Now we prove the local existence for the linear problem (2.1).

Theorem 2.3. Let the initial data $u_{0} \in \mathbf{L}^{2}$ and $f \in \mathbf{L}^{2}$. Then for any $T>0$ there exists a unique solution $u \in \mathbf{C}\left([0, T] ; \mathbf{L}^{2}\right) \cup \mathbf{C}\left((0, T] ; \mathbf{H}^{1}\right)$ of the linear initial-boundary value problem (2.1) such that

$$
\sup _{t \in(0, T]} t^{\alpha}\left\|\partial_{x}^{n} u(t)\right\|_{\mathbf{L}^{2}} \leq C \lambda
$$

provided that

$$
\lambda=\left\|u_{0}\right\|_{\mathbf{L}^{2}}+T^{1-\beta} \sup _{t \in(0, T]} t^{\beta}\|f(t)\|_{\mathbf{L}^{2}}<\infty,
$$

where $n=0,1, \alpha \in\left(\frac{n}{2}, 1\right), \beta \in(0,1)$. 
Proof. ¿From Proposition and estimates of Lemma 2.2 we have

$$
\begin{aligned}
\left\|\partial_{x}^{n} u(t)\right\|_{\mathbf{L}^{2}} \leq & t^{-\alpha}\left\|\int_{0}^{1} u_{0}(y)|x-y|^{2 \alpha-1-n} d y\right\|_{\mathbf{L}^{2}} \\
& +\left\|\int_{0}^{t} \tau^{-\alpha}(t-\tau)^{-\beta} d \tau \int_{0}^{1}(t-\tau)^{\beta} f(y, t-\tau)|x-y|^{2 \alpha-1-n} d y\right\|_{\mathbf{L}^{2}} \\
\leq & C t^{-\alpha}\left(\left\|u_{0}(y)\right\|_{\mathbf{L}^{2}}+T^{1-\beta} \sup _{t \in(0, T]} t^{\beta}\|f(\cdot, t)\|_{\mathbf{L}^{2}}\right)
\end{aligned}
$$

for $n=0,1$. So we have the estimate of the theorem. Theorem 2.3 is proved.

\section{Local Existence for the Nonlinear Problem}

In this section we prove the following result.

Theorem 3.1. Suppose that the initial data $u_{0}(x) \in \mathbf{L}^{2}$. Then there exists a unique solution

$$
u(x, t) \in \mathbf{C}\left([0, T] ; \mathbf{L}^{\infty}\right) \cup \mathbf{C}\left((0, T] ; \mathbf{H}^{1}\right)
$$

where $T>0$ depends on $\left\|u_{0}\right\|_{\mathbf{L}^{2}}$.

Proof. We prove the local existence of solutions by the contraction mapping principle. Let $u(x, t)$ be a solution of the following linear problem

$$
\left\{\begin{array}{c}
u_{t}+\mathbb{N}(w)-u_{x x}+u_{x x x}=0, t>0, x \in(0,1), \\
u(x, 0)=u_{0}(x), x \in(0,1), \\
u(0, t)=u(1, t)=u_{x}(1, t)=0, t>0,
\end{array}\right.
$$

where $\mathbb{N}(w)=w w_{x}, w$ is taken from the closed ball

$$
\mathbf{H}_{\rho}^{1}=\left\{w \in \mathbf{C}\left((0, T] ; \mathbf{H}^{1}\right) ; \sup _{t \in(0, T]} \sum_{n=0}^{1} t^{\alpha_{n}}\left\|\partial_{x}^{n} w\right\|_{\mathbf{L}^{2}} \leq \rho\right\},
$$

where $\alpha_{n} \in\left(\frac{n}{2}, 1\right)$ and satisfies the boundary condition $w(0, t)=w(1, t)=w_{x}(1, t)=0$. The initial-value problem (3.1) defines a mapping $\mathbb{M}$ by $u=\mathbb{M}(w)$ and we will show that $\mathbb{M}$ is the contraction mapping from $\mathbf{H}_{\rho}^{1}$ into itself for a sufficiently small $T>0$. Since $w \in \mathbf{H}_{\rho}^{1}$, using the estimate $\|w\|_{\mathbf{L}^{\infty}} \leq 2\|w\|_{\mathbf{L}^{2}}\left\|w_{x}\right\|_{\mathbf{L}^{2}}$, we have

$$
\sup _{t \in(0, T]} t^{\beta}\|\mathbb{N}(w)(t)\|_{\mathbf{L}^{2}} \leq C \sup _{t \in(0, T]} t^{\beta}\|w(t)\|_{\mathbf{L}^{2}}^{\frac{1}{2}}\left\|w_{x}(t)\right\|_{\mathbf{L}^{2}}^{\frac{3}{2}} \leq C \rho^{2},
$$

where $\beta=\frac{\alpha_{0}+3 \alpha_{1}}{2}<1$. Via Theorem 2.3 problem (3.1) has a unique solution $u(x, t) \in \mathbf{C}\left((0, T] ; \mathbf{H}^{1}\right)$, such that

$$
\sup _{t \in(0, T]} t^{\alpha_{n}}\left\|\partial_{x}^{n} u\right\|_{\mathbf{L}^{2}} \leq C \lambda
$$


where

$$
\lambda=\left\|u_{0}\right\|_{\mathbf{L}^{2}}+T^{1-\beta} \sup _{t \in(0, T]} t^{\beta}\|\mathbb{N}(w)(t)\|_{\mathbf{L}^{2}} .
$$

Therefore we obtain the estimate

$$
\sup _{t \in(0, T]} \sum_{n=0}^{1} t^{\alpha_{n}}\left\|\partial_{x}^{n} u\right\|_{\mathbf{L}^{2}} \leq C\left\|u_{0}\right\|_{\mathbf{L}^{2}}+C T^{1-\beta} \rho^{2} \leq \rho
$$

if $T=(2 C \rho)^{-\frac{1}{1-\beta}}=\left(4 C^{2}\left\|u_{0}\right\|_{\mathbf{L}^{2}}\right)^{-\frac{1}{1-\beta}}$ and $C\left\|u_{0}\right\|_{\mathbf{L}^{2}} \leq \frac{\rho}{2}$. Thus the mapping $\mathbb{M}$ transforms the closed ball $\mathbf{H}_{\rho}^{1}$ with a center at the origin and a radius $\rho$ into itself. Analogously we can estimate the difference

$$
\sup _{t \in(0, T]} \sum_{n=0}^{1} t^{\alpha_{n}}\left\|\partial_{x}^{n}(u-\widetilde{u})\right\|_{\mathbf{L}^{2}} \leq \frac{1}{2} \sup _{t \in(0, T]} \sum_{n=0}^{1} t^{\alpha_{n}}\left\|\partial_{x}^{n}(w-\widetilde{w})\right\|_{\mathbf{L}^{2}}
$$

for sufficiently small $T>0$. Therefore the mapping $\mathbb{M}$ is a contraction mapping in $\mathbf{H}_{\rho}^{1}$ and there exists a unique solution $u(x, t) \in \mathbf{C}\left((0, T] ; \mathbf{H}^{1}\right)$ of the initial-value problem (1.1). Theorem 3.1 is proved.

Remark 3.1. By virtue of estimate (3.2) we see that if the initial data $u_{0}$ are small, i.e. the norm $\left\|u_{0}\right\|_{\mathbf{L}^{2}} \leq \varepsilon$, where $\varepsilon>0$ is sufficiently small, then there exists $T \geq 1$, such that there exists a unique solution $u$, which is also small: $\sup _{t \in(0, T]} \sum_{n=0}^{1} t^{\alpha_{n}}\left\|\partial_{x}^{n} u\right\|_{\mathbf{L}^{2}}<C \varepsilon$.

\section{Large Time Asymptotics}

In this section we give sufficient conditions for global existence of solutions to the initial-boundary value problem (1.1) with small initial data

$$
\left\|u_{0}\right\|_{\mathbf{L}^{2}}<\varepsilon_{1}
$$

where $\varepsilon_{1}>0$ is sufficiently small.

Theorem 4.1. Suppose that the initial data $u_{0} \in \mathbf{L}^{2}$ and satisfy (4.1). Then there exists a unique solution $u$ of (1.1) such that

$$
u \in \mathbf{C}\left([0, \infty) ; \mathbf{L}^{2}\right) \cup \mathbf{C}\left((0, \infty) ; \mathbf{H}^{1}\right) .
$$

Furthermore the solution has the following asymptotics

$$
u(x, t)=A \Lambda(x) e^{-\xi_{0} t}+O\left(e^{-\left(\xi_{0}+\delta\right) t}\right)
$$

for $t \rightarrow \infty$ uniformly with respect to $x \in(0,1)$, where $\xi_{0}>0, \delta>0$, the constant $A$, the function $\Lambda(x) \in \mathbf{L}^{\infty}$ are defined below in the proof. 
Proof. According to Theorem 3.1 and Remark 3.1 we see that after some time $T \geq 1$ the solution is small in the norm $\mathbf{H}^{1}$. Therefore we can only consider the initial-boundary problem (1.1) for $t \geq 1$ with small initial data $u(x, 1)$ such that $\|u(\cdot, 1)\|_{\mathbf{H}^{1}} \leq \varepsilon_{2}$, where $\varepsilon_{2}>0$ is sufficiently small. Let us prove that

$$
e^{\xi_{0} t}\|u(t)\|_{\mathbf{H}^{1}}<\varepsilon
$$

for all $t \geq 1$, with some small $\varepsilon>0$. By the contradiction we can find a maximal interval $\left[1, T_{1}\right]$ such that the estimate

$$
e^{\xi_{0} t}\|u(t)\|_{\mathbf{H}^{1}} \leq \varepsilon
$$

is true for all $t \in\left[1, T_{1}\right]$ and estimate (4.3) is violated at time $t=T_{1}$. From (4.4) and estimates (2.31) of Lemma 2.2 we obtain for $n=0,1$

$$
\begin{aligned}
\left\|u_{x}^{(n)}(\cdot, t)\right\|_{\mathbf{L}^{2}} \leq & C e^{-\xi_{0} t}\|u(\cdot, 1)\|_{\mathbf{L}^{2}}+\int_{1}^{t} d \tau \int_{0}^{x}\left|u u_{y}(y, \tau)\right|\left\|\partial_{x}^{n} F_{1}(\cdot, y, t-\tau)\right\|_{\mathbf{L}^{\infty}} d y \\
& +\int_{1}^{t} d \tau \int_{x}^{1}\left|u u_{y}(y, \tau)\right|\left\|\partial_{x}^{n} F_{2}(\cdot, y, t-\tau)\right\|_{\mathbf{L}^{\infty}} d y \\
\leq & C \varepsilon_{2} e^{-\xi_{0} t}+C e^{-\xi_{0} t} \int_{1}^{t} e^{\xi_{0} \tau}\|u(\tau)\|_{\mathbf{L}^{2}}^{\frac{1}{2}}\left\|u_{x}(\tau)\right\|_{\mathbf{L}^{2}}^{\frac{3}{2}}\{t-\tau\}^{-\frac{3}{4}} d \tau \\
\leq & C \varepsilon_{2} e^{-\xi_{0} t}+C \varepsilon^{2} e^{-\xi_{0} t} \int_{1}^{t} e^{-\xi_{0} \tau}\{t-\tau\}^{-\frac{3}{4}} d \tau \leq C\left(\varepsilon_{2}+\varepsilon^{2}\right) e^{-\xi_{0} t}
\end{aligned}
$$

for $t \in\left[1, T_{1}\right]$. The contradiction obtained proves (4.3). Now using the estimate (4.3) and Lemma 2.2 we prove that the solution has asymptotics (4.2) for $t \rightarrow \infty$ uniformly with respect to $x>0$, where

$$
\begin{gathered}
\Lambda(x)=-\frac{\widetilde{\triangle}\left(-\xi_{0}, 1-x\right)}{\widetilde{\triangle}^{\prime}\left(-\xi_{0}, 1\right)} \\
A=\int_{0}^{1} u_{0}(y) \widetilde{\triangle}\left(-\xi_{0}, y\right) d y+\int_{0}^{\infty} e^{\xi_{0} \tau} d \tau \int_{0}^{1} u(y, \tau) u_{y}(y, \tau) \widetilde{\triangle}\left(-\xi_{0}, y\right) d y .
\end{gathered}
$$

Indeed, due to asymptotics (2.30) of Lemma 2.2 we have

$$
u(x, t)=A \Lambda(x) e^{-\xi_{0} t}+R(x, t),
$$

where in view of (4.3) we have

$$
\begin{aligned}
|R(x, t)| \leq & e^{-\left(\xi_{0}+\delta\right) t}\|u(\cdot, 1)\|_{\mathbf{H}^{1}}+C \int_{1}^{t} e^{-\left(\xi_{0}+\delta\right)(t-\tau)} d \tau \int_{0}^{1}\left|u u_{y}\right| d y \\
& +e^{-\xi_{0} t}|\Lambda(x)| \int_{t}^{\infty} e^{\xi_{0} \tau} d \tau \int_{0}^{1}\left|u u_{y}\right|\left|\widetilde{\triangle}\left(-\xi_{0}, y\right)\right| d y \\
= & O\left(e^{-\left(\xi_{0}+\delta\right) t}\right)
\end{aligned}
$$

for all $t \geq 1$, where $\delta>0$. Theorem 4.1 is proved. 


\section{$5 \quad$ Large Initial Data}

We consider the initial-boundary value problem (1.1) with any initial data $\left\|u_{0}\right\|_{\mathbf{L}^{2}} \leq C$. Multiplying equation (1.1) by $u$ and integrating with respect to $x \in(0,1)$ we get

$$
\frac{d}{d t}\|u\|_{\mathbf{L}^{2}}^{2}+2 \int_{0}^{1}\left(u^{2} u_{x}-u u_{x x}+u u_{x x x}\right) d x=0 .
$$

We have

$$
\begin{gathered}
\int_{0}^{1} u^{2} u_{x} d x=\left.\frac{1}{3} u^{3}\right|_{0} ^{1}=0 \\
\int_{0}^{1} u u_{x x} d x=\left.u u_{x}\right|_{0} ^{1}-\int_{0}^{1} u_{x}^{2} d x=-\int_{0}^{1} u_{x}^{2} d x \\
\int_{0}^{1} u u_{x x x} d x=\left.u u_{x x}\right|_{0} ^{1}-\left.\frac{1}{2} u_{x}^{2}\right|_{0} ^{1}=\frac{1}{2} u_{x}^{2}(0, t)
\end{gathered}
$$

in view of the boundary data, hence

$$
\frac{d}{d t}\|u\|_{\mathbf{L}^{2}}^{2}+2 \int_{0}^{1} u_{x}^{2} d x+u_{x}^{2}(0, t)=0 .
$$

Integration with respect to $t>0$ yields

$$
\|u(t)\|_{\mathbf{L}^{2}}+2 \int_{0}^{t}\left\|u_{x}(\tau)\right\|_{\mathbf{L}^{2}}^{2} d \tau \leq\left\|u_{0}\right\|_{\mathbf{L}^{2}}
$$

for all $t \in(0, \infty)$. We see that the norm $\|u(t)\|_{\mathbf{L}^{2}} \leq\left\|u_{0}\right\|_{\mathbf{L}^{2}}$ for all $t \geq 0$. By the standard continuation process via Theorem 3.1 we obtain that there exists a unique global solution $u \in$ $\mathbf{C}\left((0, \infty) ; \mathbf{H}^{1}\right)$ since the existence time $T$ depends only on $\left\|u_{0}\right\|_{\mathbf{L}^{2}}$. Moreover we see that for any $\varepsilon>0$ there exists a time $T>0$ such that $\left\|u_{x}(T)\right\|_{\mathbf{L}^{2}}^{2}<\varepsilon$. By the inequality $u^{2}(x, T)=$ $2 \int_{0}^{x} u u_{y} d y \leq 2\|u\|_{\mathbf{L}^{2}}\left\|u_{x}\right\|_{\mathbf{L}^{2}}$ we see that the norm $\|u(T)\|_{\mathbf{L}^{\infty}}$, and hence the norm $\|u(T)\|_{\mathbf{L}^{2}}$, are also small by the estimate $\|u(T)\|_{\mathbf{L}^{2}} \leq\|u(T)\|_{\mathbf{L}^{\infty}}$. Then we consider the initial-boundary value problem (1.1) for $t \geq T$ and apply Theorem 4.1 whence the result of Theorem 1.1 follows. Theorem 1.1 is proved.

Acknowledgments. The work of One of the authors (E.I.K.) is partially supported by CONACYT.

Received: September, 2008. Revised: October, 2009.

\section{References}

[1] Aikawa, H. and Hayashi, N., Holomorphic solutions of semilinear heat equations, Complex Variables, 16 (1991), pp. 115-125. 
[2] Aikawa, H., Hayashi, N. and Saitoh, S., The Bergman space on a sector and the heat equation, Complex Variables, 15 (1990), pp. 27-36.

[3] Amick, C.J., Bona, J.L. And Schonbek, M.E., Decay of solutions of some nonlinear wave equations, J. Diff. Eq. 81 (1989), pp. 1-49.

[4] Biller, P., Asymptotic behavior in time of solutions to some equations generalizing the Korteweg-de Vries-Burgers equation, Bull. Polish. Acad. Sc. Math., 32 (1984), pp. 275-282.

[5] Bona, J.L. And Luo, L., More results on the decay of the solutions to nonlinear dispersive wave equations, Discrete and Continuous Dynamical Systems, 1 (1995), pp. 151-193.

[6] Dix, D.B., The dissipation of nonlinear dispersive waves; the case of asymptotically weak nonlinearity, Comm. P.D.E., 17 (1992), pp. 1665-1693.

[7] Hayashi, N., Kaikina, E.I. and Manzo, R., Local and global existence of solutions to the nonlocal Whitham equation on half-line, Nonlinear Analisys, 48 (2002), pp. 53-75.

[8] Hayashi, N., Kaikina, E.I. And Paredez, F.R., Boundary-value problem for the Korteweg-de Vries-Burgers type equation, Nonlinear Differential Equations and Applications, 8, No. 4 (2001), pp. 439-463.

[9] Hayashi, N., Kaikina, E.I. And Shishmarev, I.A., Asymptotics of Solutions to the Boundary-Value Problem for the Korteweg-de Vries-Burgers equation on a Half-Line, Journal of Mathematical Analysis and Applications, 265 (2002), No. 2, pp. 343-370.

[10] Kaikina, E.I., Naumkin, P.I. and Shishmarev, I.A., Asymptotic behavior for large time of solutions to the nonlinear nonlocal Schrödinger equation on a half-line, SUT Journal of Mathematics, 35 (1) (1999), pp. 37-79.

[11] Naumkin, P.I. and Shishmarev, I.A., Asymptotic behavior for large time of solutions of Korteweg-de Vries equation with dissipation,Differential Equations, 29 (1993), pp. 253-263.

[12] Naumkin, P.I. And Shishmarev, I.A., Asymptotic relationship as $t \rightarrow \infty$ between solutions to some nonlinear equations, Differential Equations, 30 (1994), pp. 806-814.

[13] Shishmarev, I.A., Tsutsumi, M. and Kaikina, E.I., Asymptotics in time for the nonlinear nonlocal Schrödinger equations with a source, J. Math. Soc. Japan, 51 (1999), pp. 463-484. 